Correction

\title{
Correction: Naudts, J. Quantum Statistical Manifolds. Entropy 2018, 20, 472
}

\section{Jan Naudts 1}

Departement Fysica, Universiteit Antwerpen, Universiteitsplein 1, 2610 Wilrijk Antwerpen, Belgium; jan.naudts@uantwerpen.be

Received: 6 September 2018; Accepted: 12 October 2018; Published: 17 October 2018

Abstract: Section 4 of "Naudts J. Quantum Statistical Manifolds. Entropy 2018, 20, 472" contains errors. They have limited consequences for the remainder of the paper. A new version of this Section is found here. Some smaller shortcomings of the paper are taken care of as well. In particular, the proof of Theorem 3 was not complete, and is therefore amended. Also, a few missing references are added.

\section{Theorem 1.}

\section{Theorem 2.}

\section{Corrections in Section 3}

The display on top of page 5 should read

$$
\begin{aligned}
\left\|f_{\rho, K}\right\| & =\sup _{A \in \mathcal{A}}\left\{f_{\rho, K}(A):\|A\| \leq 1\right\} \\
& =\sup _{A \in \mathcal{A}}\left\{\left(\pi(A) K \Omega_{\rho}, \Omega_{\rho}\right):\|A\| \leq 1\right\} \\
& =\left\||K|^{1 / 2} \Omega_{\rho}\right\|^{2} \\
& \leq\left\||K|^{1 / 2}\right\|^{2}=\|K\| .
\end{aligned}
$$

The operator $K$ is replaced by $|K|$ because $K$ need not be positive.

The sentence "This is a prerequisite for proving in the next Theorem that this map is the Fréchet derivative of the chart $\xi \rho$." should read "This is a prerequisite for proving in the next Theorem that this map is the Fréchet derivative of the inverse of the chart $\xi_{\rho} . "$

The proof of the following Theorem is amended.

Theorem 3. The inverse of the map $\xi_{\rho}: \mathbb{M} \mapsto \mathcal{B}_{\rho}$, defined in Theorem 2, is Fréchet-differentiable at $\omega=\omega_{\rho}$. The Fréchet derivative is denoted $F_{\rho}$. It maps $K$ to $f_{\rho, K}$, where the latter is defined by (10).

Proof. Let $K=\xi_{\rho}\left(\omega_{\sigma}\right)$. One calculates

$$
\begin{aligned}
\left\|\omega_{\sigma}-\omega_{\rho}-F_{\rho} K\right\| & =\sup _{A \in \mathcal{A}}\left\{\left|\omega_{\sigma}(A)-\omega_{\rho}(A)-F_{\rho} K(A)\right|:\|A\| \leq 1\right\} \\
& =\sup _{A \in \mathcal{A}}\left\{\left|\left(\pi(A) \Omega_{\rho},\left[e^{K-\alpha(K)}-\mathbb{I}-K\right] \Omega_{\rho}\right)\right|:\|A\| \leq 1\right\} \\
& \leq\left\|e^{K-\alpha(K)}-\mathbb{I}-K\right\| \\
& \leq|\alpha(K)|+\mathrm{o}(\|K-\alpha(K)\|) .
\end{aligned}
$$

Note that

$$
|\alpha(K)| \leq \log \left\|e^{K}\right\| \leq\|K\|
$$


and

$$
\|K-\alpha(K)\| \leq 2|| K \| .
$$

In addition, if $\|K\|<1$ then one has

$$
\alpha_{\rho}(K) \leq \log \left(1+\left\|K \Omega_{\rho}\right\|^{2}\right) \leq\left\|K \Omega_{\rho}\right\|^{2} .
$$

This holds because $\lambda \leq 1$ implies $\exp (\lambda) \leq 1+\lambda+\lambda^{2}$. One concludes that (11) converges to 0 faster than linearly as $\|K\|$ tends to 0 . This proves that $F_{\rho} K$ is the Fréchet derivative of $\xi_{\rho}\left(\omega_{\sigma}\right) \mapsto \omega_{\sigma}$ at $\sigma=\rho$.

\section{New Version of Section 4}

Propositions 1 and 2 of [1] are not correct. This only has consequences for one sentence in the Introduction of [1] and for the results reported in Section 4 of [1]. The text in the Introduction "Next, an atlas is introduced which contains a multitude of charts, one for each element of the manifold. Theorem 4 proves that the manifold is a Banach manifold and that the cross-over maps are linear operators." should be changed to "Next, an atlas is introduced which contains a multitude of charts, one for each element of the manifold. Theorem 4 proves that the manifold is a Banach manifold and that the cross-over maps are continuous."

A new version of Section 4 follows below:

\section{The Atlas}

Following the approach of Pistone and collaborators [1,3,4,24], we build an atlas of charts $\xi_{\rho}$, one for each strictly positive density matrix $\rho$. The compatibility of the different charts requires the study of the cross-over map $\xi_{\rho_{1}}(\sigma) \mapsto \xi_{\rho_{2}}(\sigma)$, where $\rho_{1}, \rho_{2}, \sigma$ are arbitrary strictly positive density matrices.

Simplify notations by writing $\xi_{1}$ and $\xi_{2}$ instead of $\xi_{\rho_{1}}$, respectively $\xi_{\rho_{2}}$. Similarly, write $\Omega_{1}$ and $\Omega_{2}$ instead of $\Omega_{\rho_{1}}$, respectively $\Omega_{\rho_{2}}$, and $F_{1}, F_{2}$ instead of $F_{\rho_{1}}$, respectively $F_{\rho_{2}}$.

\section{Proposition 1. RETRACTED}

Continuity of the cross-over map follows from the continuity of the exponential and logarithmic functions and from the following result.

Proposition 2. Fix strictly positive density matrices $\rho_{1}$ and $\rho_{2}$. There exists a linear operator $Y$ such that for any strictly positive density matrix $\sigma$ and corresponding positive operators $X_{1}, X_{2}$ in the commutant $\mathcal{A}^{\prime}$ one has $X_{2}=Y X_{1} Y^{*}$

Proof. Using the notations of the Appendix of [1], one has

$$
X_{i}=J_{i}\left(\rho_{i}^{-1 / 2} \sigma \rho_{i}^{-1 / 2} \otimes \mathbb{I}\right) J_{i}^{*}, \quad i=1,2 .
$$

Note that the isometry $J$ depends on the reference state with density matrix $\rho$. Therefore, it carries an index $i$. The above expression for $X_{i}$ implies that

$$
X_{2}=Y X_{1} Y^{*} \quad \text { with } \quad Y=J_{2}\left(\rho_{2}^{-1 / 2} \rho_{1}^{1 / 2} \otimes \mathbb{I}\right) J_{1}^{*} .
$$


Theorem 4. The set $\mathbb{M}$ of faithful states on the algebra $\mathcal{A}$ of square matrices, together with the atlas of charts $\xi_{\rho}$, where $\xi_{\rho}$ is defined by Theorem 1, is a Banach manifold. For any pair of strictly positive density matrices $\rho_{1}$ and $\rho_{2}$, the cross-over map $\xi_{2} \circ \xi_{1}^{-1}$ is continuous.

Proof. The continuity of the map $X_{1} \mapsto X_{2}$ follows from the previous Proposition. The continuity of the maps $K_{1} \mapsto X_{1}$ and $X_{2} \mapsto K_{2}$ follows from the continuity of the exponential and logarithmic functions and the continuity of the function $\alpha$.

\section{Corrections in Section 9}

In the proof of Proposition 4 , the symbol $\Omega_{\rho}$ is missing five times in obvious places. It has been added.

\section{Added References}

In the overview of papers devoted to the study of the quantum statistical manifold in the finite-dimensional case, the references [2,3] should be added. A quantum version of the work of Pistone and Sempi [4], alternative to [5], is found in [6]. Reference [7] to the work of Ciaglia et al. has been updated.

\section{References}

1. Naudts, J. Quantum Statistical Manifolds. Entropy 2018, 20, 472. [CrossRef]

2. Petz, D.; Sudar, C. Geometries of quantum states. J. Math. Phys. 1996, 37, 2662-2673. [CrossRef]

3. Jenčová, A. Geometry of quantum states: Dual connections and divergence functions. Rep. Math. Phys. 2001, 47, 121-138. [CrossRef]

4. Pistone, G.; Sempi, C. An infinite-dimensional structure on the space of all the probability measures equivalent to a given one. Ann. Stat. 1995, 23, 1543-1561. [CrossRef]

5. Streater, R.F. Quantum Orlicz spaces in information geometry. Open Syst. Inf. Dyn. 2004, 11, 359-375. [CrossRef]

6. Jenčová, A. A construction of a nonparametric quantum information manifold. J. Funct. Anal. 2006, 239, 1-20. [CrossRef]

(C) 2018 by the author. Licensee MDPI, Basel, Switzerland. This article is an open access article distributed under the terms and conditions of the Creative Commons Attribution (CC BY) license (http:/ / creativecommons.org/licenses/by/4.0/). 